\title{
Study on Fuzzy Catastrophe Risk Model Based on Fuzzy Theory
}

\author{
Xiaoxia Zhu \\ School of Sciences, Hebei University of Science and Technology, Shijiazhuang \\ (China) \\ zhuxiaoxia66@126.com
}

\begin{abstract}
In this paper, we analyzed the essential meaning of the catastrophe risk on the basis of many results that is the bottleneck of the catastrophe risk analysis is complex. One way to resolve or alleviate this problem is to analyze the risk from the viewpoint of fuzzy logicfuzzy catastrophic risk (FCR) analysis. We propose the concept of synthetic effect, present its axiomatic foundation, and further establish a FCR model based on synthetic effect as well as the general solution model. Finally, we specify the model by considering catastrophe risk in Shanghai. The results indicate that the method is not only accommodates the existing fuzzy decision-making methods, but also successfully incorporates the decision preference into the optimization process. Therefore, the FCR model can be widely used in many fields such as complex systems optimization and decision-making.
\end{abstract}

Keywords: Catastrophe Risk; Fuzzy Risk; Fuzzy Sets; Synthetic Effect

\section{Introduction}

The frequency and the loss of global catastrophe events have risen sharply since the 1990s. Many countries and regions face the threats of all kinds of catastrophe risk, which may have increasingly strong impact on our human society, Such as 2008 earthquake in China, 2011 earthquake and tsunami in Japan, and so on. Catastrophes are inevitable, but the loss can be reduced through the prevention and improvement of human anti-disaster ability, thus the damage and influence of catastrophes on the human society can be effectively reduced and which depends on the awareness of people's catastrophe risk (Tian, 2009). So, it has a very important significance to reduce damage and loss and to improve the economic benefit of human society by the means of scientific and reasonable description of catastrophe risk.

At present, it hasn't uniform definition of catastrophe risk in China and other Countries, but the understanding of catastrophe risk characteristics has been a general consensus. UNDP (1991) given the definition of catastrophe risk, that was expected loss values about lives, property and economic activities by catastrophe within a certain area and a given time period, the degree of catastrophe risk was expressed as"Risk Degree =Hazard DegreexVulnerability" (Maskrey, 1989).Standard \&poor's (1999) defined catastrophe as a risk event or a series of risk events which caused more than 5 million dollars losses, and this risk is called catastrophe risk. In this paper, we believe that" catastrophe risk" is the uncertainty of loss which caused by catastrophe, including the possibility of disaster, the degree of hazard and the degree of loss, and so on. However, "catastrophe" includes natural disasters and man-made disasters that are large-scale and has particularly serious destruction, and has a significant impact on economic development and social stability in regional and national. Compared to other risks of the human society, catastrophe risk has typical characteristics as following: First, complex causes, and localized significantly. Second, huge potential losses, and broad correlation. Third response to catastrophic risk tends to super plan, and need public power enter. 
In addition, Catastrophe evaluation is an important part in the study of disaster and is the basis of hazard prediction, prevention and cure research and compensation. Catastrophe evaluation can be divided into risk assessment, loss evaluation, and evaluation of ecological environment, disaster prevention and disaster reduction benefit evaluation, etc. (Huang,2005). Among them, Catastrophe risk assessment is the basic research to prevent catastrophe, control and reduce the risk of catastrophe, on induced catastrophe factor and pregnant disaster environment angle, through the analysis of frequency and intensity of natural phenomenon which lead to catastrophe happens, Establish and save historical catastrophe record, Draw zoning maps of all sorts of disaster risk, to estimate the vulnerability and the potential impact(Thierry,2008).In 1987 the United States federal emergency department (FEMA) entrust application technology commission (ATC) to execute the ATC-13 plan, the plan systematic collect the experts and scholars views, Established the most complete earthquake disaster assessment material at the time. The program results provide of all kinds of buildings, public facilities, the economic loss and vulnerability assessment information of casualties. After the plan, because of the requirement of United States banking, insurance and government departments, stimulate the earthquake risk assessment system upgrade and development and promote the born of catastrophe risk assessment model(Tian,2009).From the existing catastrophe risk assessment results, the assessment method of catastrophe risks can be divided into four categories, as following (Sun, 2010).

\subsection{Data Analysis Method}

Data analysis method is mainly to use the method of mathematical analysis, Statistical analysis of a natural phenomenon information and documentation, and to get the disaster statistical rule.

\subsection{Simulation Method}

This method is based on the study of catastrophe theory, through the experimental method, simulation the formation mechanism of disasters, transmission mechanism, and the role of hazard-formative factors and the results of disasters, in this way, Can purify the influence of hazard-formative factors, Rule out interference of confounding factors, Reveal the forming mechanism of the catastrophe, provide the basis for the catastrophe risk prediction and prevention and control.

\subsection{The Mathematic Model and Method}

Mathematical model is used to evaluate the risk of catastrophe. At present, these already developed models which can be used on the catastrophe risk assessment including: fuzzy comprehensive evaluation, neural network, probability model, data envelopment, Information entropy, gray system, dynamics model, Information diffusion model, etc.

\subsection{Remote Sensing GIS Method}

Remote sensing technology is mainly used on the catastrophe and the investigation of the catastrophe of the dynamic monitoring, GIS is mainly used on data management and the prediction.

Based on above analysis, we find that the existing definitions are based on random uncertainty of the catastrophe risk losses. First, the characteristics of the catastrophe risk indicate that the catastrophe has small probability and large losses, so it is difficult to quantitatively predict the possibilities and losses level of the future catastrophic events through the historical data and statistical actuarial method. Second, Because of highly inaccurate information and its asymmetry, we often assess the possibilities and results of 
the catastrophic risk through ourselves perception when we evaluate the catastrophic risk, the assessment results are often belong to qualitative language, however these languages are fuzziness. The many shortcoming also exist in the present research results about model and method of the catastrophic risk, in the following: First, Most of them are focused on single species disaster, Comprehensive multi-hazard and composite hazard risk assessment study is also less, the timeliness and accuracy cannot meet the actual needs. Second, Most of assessment model is to catastrophe of hazard-formative factors, risk exposure, vulnerability. Third, on fuzzy comprehensive assessment of the risk of catastrophe is little research.

In this paper, the followings are the aspects discussed: First, we draw fuzzy theory into this paper, and establish the fuzzification method for language type and Real number, and particularly propose the weighted fuzzification method for family of interval information. Second, we put forward the concept of synthetic effect function for describing fuzzy information processing, establish concentrated quantification method of fuzzy information with wide application. Third, we establish comprehensive evaluation model for multicatastrophe risks or composite catastrophe risk abase on synthetic effect function. Finally, we also uses this model to evaluate the catastrophe risk of Shanghai in China. The results indicate that the model is not only feasibility, reliability and robustness, but also has some advantages which are not in other risks analysis technique.

\section{Theoretical bases}

The fuzziness is a common phenomenon in real world. In1965, Zadeh (American cyber-netics expert) proposed the concept of the fuzzy set and established fuzzy set theory, which provide a new effective tool for the description and processing of uncertain information, and these made many theoretical researches and the application results in the field of fuzzy control, artificial intelligence, expert systems, etc(Zadeh ,1965, Zimmermann ,1991).

\subsection{Fuzzy number and its Transformation rules}

\subsubsection{Fuzzy number}

Fuzzy numbers, with the feature of both fuzzy sets and numbers, are the most common tool for describing fuzzy information in real problems. In this part, we introduce the concept of fuzzy number and their basic operations.

In the following, let $R$ be the real number field, $F(R)$ the family of all fuzzy sets over $R$. For any $A \in F(R)$, the membership function of $A$ is written as $A(x)$, the $\lambda$ cuts of $A$ as $A_{\lambda}=\{x \mid A(x) \geq \lambda\}$, and the support set of $A$ as supp $A=\{x \mid A(x)>0\}$. In what following, we introduce the definition of fuzzy number and its basic operation properties.

Definition $1 A \in F(R)$ is called a fuzzy number if it satisfies the following conditions:

1) For any given $\lambda \in(0,1], A_{\lambda}$ are closed intervals; 2) $A_{1}=\{x \mid A(x)=1\} \neq \varphi$; 3) supp A is bounded. The class of all fuzzy numbers is called fuzzy number space, which is denoted by E1. In particular, if there exists $a, b, c \in R$ such that $A(x)=(x-a) /(b-a)$ for each $x \in[a, b), A(b)=1, A(x)=(x-c) /(b-c)$ for each $x \in(b, c]$, and $A(x)=0$ for each $x \in(-\infty, a) \cup(c,+\infty)$, then we say that $\mathrm{A}$ is a triangular fuzzy number(For short,TFN), and written as $A=(a, b, c)$ for short (Goetschel,1983).

For $A \in E^{1}$, it is easily to see that the closure of supp $A$ is closed interval, in what follows we denote the closure of supp $A$ by $A_{0}$. By Definition 1, we can prove that $A_{\lambda}=[a+(b-a) \lambda, c-(c-b) \lambda]$ for any $A=(a, b, c)$ and $\lambda \in[0,1]$.

Obviously, if we regard real number $a$ as a fuzzy set whose membership function is $a(x)=1$ for $x=a$ and $a(x)=0$ for each $x \neq a$, then fuzzy number can be thought 
as an extension of real number, so fuzzy numbers possess the properties of both numbers and sets, which is the most wide description of fuzzy information in many practical domains. For the arithmetic operation, we have the following conclusion:

Theorem 1 Let $A, B \in E^{1}, k \in \mathrm{R}, f(x, y)$ be a continuous binary function, $A_{\lambda}, B_{\lambda}$ be the $\lambda$-cuts of $A$ and $B$, respectively. Then $f(A, B) \in E^{1}$, and $(f(A, B))_{\lambda}=f\left(A_{\lambda}, B_{\lambda}\right)$ for any $\lambda \in(0,1]$. In particular, we have: 1) $A+B=B+A, A-B=B-A, k(A \pm B)=k A \pm k B ; 2)$ For $A=\left(a_{1}, b_{1}, c_{1}\right), B=\left(a_{2}, b_{2}, c_{2}\right)$, $A+B=\left(a_{1}+a_{2}, b_{1}+b_{2}, c_{1}+c_{2}\right), A-B=\left(a_{1}-c_{2}, b_{1}-b_{2}, c_{1}-a_{2}\right), k A=\left(k a_{1}, k b, k c_{1}\right)$ for any $k \geqslant 0$, and $k A=\left(k c_{1}, k b_{1}, k a_{1}\right)$ for any $k<0$. Here, $f\left(A_{\lambda}, B_{\lambda}\right)=\left\{f(x, y) \mid x \in A_{\lambda}, y \in B_{\lambda}\right\}$.

Fuzzy numbers have many good analytical properties. For details, please see ref (Diamond, 1994)

\subsubsection{Fuzzification Rules}

The TFN, with better structure and operation properties, is the mostly used fuzzy information description method. In the assessment processing of catastrophe risk, we may need to convert language type, real number and interval number into TFN.So, we will give transformation rules as following.

(1) Fuzzification rules for Language type

To index systems of language type, we will use TFN for Language scale as shown in Table 1.

Table 1. Language Indicators and TFN Corresponding Relationship

\begin{tabular}{|c|c|c|c|c|c|}
\hline Types & & Classificat & of Language ind & cators & \\
\hline 1 & Very larger & larger & General & Small & Very Small \\
\hline 2 & Very Good & Good & Average & Poor & Very Poor \\
\hline 3 & Very High & High & middle & Low & Very Low \\
\hline $\begin{array}{l}\text { Exact } \\
\text { values }\end{array}$ & 5 & 4 & 3 & 2 & 1 \\
\hline TFN & $(0.8,0.9,1.0)$ & $(0.6,0.7,0.8)$ & ) $\quad 0.4,0.5,0.6$ & )$^{(0.2,0.3,0.4}$ & $(0,0.1,0.2)$ \\
\hline
\end{tabular}

(2) Fuzzification rules for Real number

In the assessment processing of catastrophe risk, some index systems data need to be obtained by Statistics or computing. In order to adapt the processing method of data in this paper, we must converted them into TFN, such as, real number $T$ may be converted into TFN $(\tau, \tau, \tau)$.

(3) Fuzzification rules for Interval number

Interval is an effective data processing method between qualitative and quantitative aspect. Under some consciousness, how to transform a family of interval into fuzzy information is a hot research in many fields such as management science, optimization decision. In the following, we will give a weighted fuzzification method of interval number.

Definition2 Let the weight of $\left[a_{i}, b_{i}\right]$ be $w_{i} \in(0,1)$ in data integration, and $\sum_{i=1}^{m} w_{j}=1, \theta \in[0,1], i=1,2, \cdots, m$. Denote $c_{i}=a_{i}+\theta\left(b_{i}-a_{i}\right)$, then 


$$
c=\sum_{i=1}^{m} w_{i} c_{i}
$$

is called $\theta$-weighted fuzzification principal value of the family of $\left\{\left[a_{i}, b_{i}\right]\right\}_{i=1}^{m}$. If

$$
a=\sum_{i=1}^{m} w_{i} a_{i}, b=\sum_{i=1}^{m} w_{i} b_{i},
$$

then triangular fuzzy number $(a, c, b)$ is called $\theta$-weighted equilibrium fuzzification value of the family of $\left\{\left[a_{i}, b_{i}\right]\right\}_{i=1}^{m}$, denoted by $F\left(\left\{\left[a_{i}, b_{i}\right]\right\}_{i=1}^{m},\left\{w_{i}\right\}_{i=1}^{m}, \theta, \Sigma\right)$ for short. If

$$
a=\min _{1 \leqslant i \leqslant m} a_{i}, b=\sum_{i=1}^{m} w_{i} b_{i},
$$

then triangular fuzzy number $(a, c, b)$ is called $\theta$-weighted minimum fuzzification value of the family of $\left\{\left[a_{i}, b_{i}\right]\right\}_{i=1}^{m}$, denoted by $F\left(\left\{\left[a_{i}, b_{i}\right]\right\}_{i=1}^{m},\left\{w_{i}\right\}_{i=1}^{m}, \theta, \Sigma_{\text {min }}\right)$ for short. If

$$
a=\sum_{i=1}^{m} w_{i} a_{i}, b=\max _{1 \leq i \leq m} b_{i},
$$

then triangular fuzzy number $(a, c, b)$ is called $\theta$-weighted maximum fuzzification value of the family of $\left\{\left[a_{i}, b_{i}\right]\right\}_{i=1}^{m}$, denoted by $F\left(\left\{\left[a_{i}, b_{i}\right]\right\}_{i=1}^{m},\left\{w_{i}\right\}_{i=1}^{m}, \theta, \Sigma^{\max }\right)$ for short. If

$$
a=\min _{1 \leqslant i \leqslant n} a_{i}, b=\min _{1 \leqslant i \leqslant n} b_{i},
$$

then triangular fuzzy number $(a, c, b)$ is called $\theta$-weighted Max-Min fuzzification value of the family of $\left\{\left[a_{i}, b_{i}\right]\right\}_{i=1}^{m}$, denoted by $F\left(\left\{\left[a_{i}, b_{i}\right]\right\}_{i=1}^{m},\left\{w_{i}\right\}_{i=1}^{m}, \theta, \Sigma_{\min }^{\max }\right)$ for short.

By using the algebraic operation of fuzzy number, we have $F\left(\left\{\left[a_{i}, b_{i}\right]\right\}_{i=1}^{m},\left\{w_{i}\right\}_{i=1}^{m}, \theta, \Sigma\right)=$

$\sum_{i=1}^{m} w_{i} F\left(\left[a_{i}, b_{i}\right], \theta\right)$,

which shows $\theta$-weighted fuzzification method is a linear weighting method, and (3), (4), (5) are nonlinear weighting methods with a certain tendency.

In Definition2, 1) when $m=1$, the above four fuzzification results are same (denoted by $F([a, b], \theta)$ for short); when $m>1$, each fuzzification result maybe have greater difference; 2) $\theta$ is risk parameter reflecting fuzzification feature:a) $\theta=0.5$ corresponds to neutral decision; b) $\theta<0.5$ corresponds to conservative decision, and the smaller $\theta$ is, the greater the conservative degree is; c) $\theta>0.5$ corresponds to optimism decision, and the greater $\theta$ is, the greater the optimism degree is; for example, when $\theta$ is $0,0.25,0.5,0.75,1$ respectively, the corresponding $\theta$-weighted fuzzification results is shown as Figure 1.

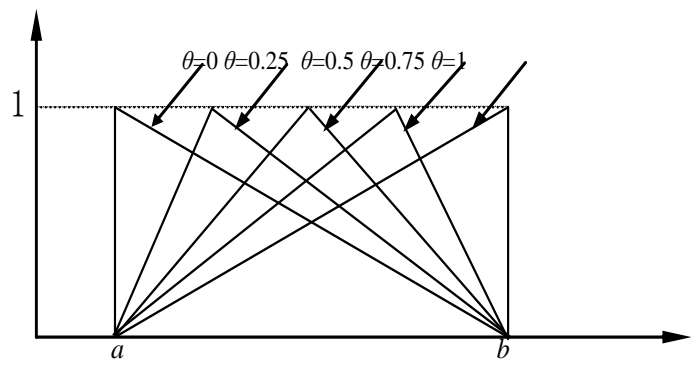

Figure 1.The visual explanation of fuzzification based on different risk value

\section{2. Fuzzy Information Standardization Rules}

In the fuzzy multiple attribute evaluation, Need to put multiple attribute together to evaluate. Because of each attribute has different dimension, different Unit, different Orders of magnitude, different Evaluation standard, can't directly use the original evaluation value to comprise and schedule. In order to eliminate the 
differences between each attribute to the influence of the evaluation results, need to standardize the original assessed value. In Literature (Chen, 2012, Zhang, 2009) the standardization formula is given. In all evaluation indexes, let $I_{1}$ shows the efficiency index collection, $I_{2}$ shows the type cost index collection, then Standardized formula is as follows:

$$
\left(I_{1}\right)\left\{\begin{array} { l } 
{ b _ { i j } ^ { L } = a _ { i j } ^ { L } / \sqrt { \sum _ { i = 1 } ^ { m } ( a _ { i j } ^ { L } ) ^ { 2 } } } \\
{ b _ { i j } ^ { M } = a _ { i j } ^ { M } / \sqrt { \sum _ { i = 1 } ^ { m } ( a _ { i j } ^ { M } ) ^ { 2 } } ( j \in I _ { 1 } ) , ( I _ { 2 } ) } \\
{ b _ { i j } ^ { U } = a _ { i j } ^ { U } / \sqrt { \sum _ { i = 1 } ^ { m } ( a _ { i j } ^ { U } ) ^ { 2 } } }
\end{array} \left\{\begin{array}{l}
b_{i j}^{L}=\left(1 / a_{i j}^{L}\right) / \sqrt{\sum_{i=1}^{m}\left(1 / a_{i j}^{L}\right)^{2}} \\
b_{i j}^{M}=\left(1 / a_{i j}^{M}\right) / \sqrt{\sum_{i=1}^{m}\left(1 / a_{i j}^{M}\right)^{2}}\left(j \in I_{2}\right) \\
b_{i j}^{U}=\left(1 / a_{i j}^{U}\right) / \sqrt{\sum_{i=1}^{m}\left(1 / a_{i j}^{U}\right)^{2}}
\end{array}\right.\right.
$$

Here, $\left(a^{L}, a^{u}, a^{u}\right)$ Said respectively a triangular fuzzy number left value, median and right value.

\subsection{Compound Quantification Description of Fuzzy Number}

Ordering fuzzy numbers, a main part of the theory of fuzzy numbers, is the key to fuzzy optimization problems. Up to now, the usually procedure is to map fuzzy numbers by an appropriate transformation into a real number and thus realize a comparison and ordering of fuzzy numbers. To establish a general quantification of fuzzy information, we introduce the following definition $(\mathrm{Li}, 2012)$.

Definition3 We call $L(\lambda):[0,1] \rightarrow[a, b] \subset[0, \infty)$ a level effect function, if $L(\lambda)$ is piecewise continuous and monotone non-decreasing. For $A \in E^{1}$, let

$$
\begin{gathered}
I_{L}(A)=\frac{1}{2 L^{*}} \int_{0}^{1} L(\lambda)(\underline{a}(\lambda)+\bar{a}(\lambda)) \mathrm{d} \lambda, \\
U_{L}(A)=\int_{0}^{1} L(\lambda)(\bar{a}(\lambda)-\underline{a}(\lambda)) \mathrm{d} \lambda .
\end{gathered}
$$

Then $I_{L}(A)$ is called the centralized quantification principle value of $A$, and $U_{L}(A)$ is the dispersion of $A$. When $L^{*}=0$, we define $I_{L}(A)=[\underline{a}(1)+\bar{a}(1)] / 2$, and $U_{L}(A)=\bar{a}(1)-\underline{a}(1)$. Here, $A_{\lambda}=[\underline{a}(\lambda), \bar{a}(\lambda)]$ is the $\lambda$-cuts of $A, L^{*}=\int_{0}^{1} L(\lambda) \mathrm{d} \lambda$.

Theorem2 Let $A, B \in E^{1}, a, k \in \mathrm{R}$. Then: 1) $I_{L}(A \pm B)=I_{L}(A) \pm I_{L}(B), U_{L}(A \pm B)=$ $U_{L}(A)+U_{L}(B)$; 2) $\left.I_{L}(k A)=k I_{L}(A), U_{L}(k A)=|k| U_{L}(A) ; 3\right) \quad I_{L}(a+A)=a+I_{L}(A)$, $U_{L}(a+A)=U_{L}(A)$.

This theorem can be obtained by the definition of fuzzy number and the properties of integral.

Theorem3 For $A=(a, b, c), a \in[0, \infty)$, when $L(\lambda)=\lambda^{a}$, we have

$$
I_{L}(A)=\frac{a+2 a b+c}{2(a+2)}, U_{L}(A)=\frac{c-a}{(a+1)(a+2)} .
$$

Definition4 $A$ continuous function $S(x, y):(-\infty,+\infty) \times[0,+\infty) \rightarrow(-\infty,+\infty)$ is called a maximum synthesizing effect function, if it satisfies: 1) $S(x, y)$ is monotone nondecreasing on $x$ for any $y \geqslant 0 ; 2) S(x, y)$ is monotone non-increasing on $y$ for any $x \in(-\infty, \infty) ; 3) S(x, y) \leqslant x, S(x, 0)=x$.

If we regard $x$ and $y$ as $I_{L}(A)$ and $U_{L}(A)$ of $A$ respectively, then

$$
S_{L}(A)=S\left(I_{L}(A), U_{L}(A)\right)
$$


is a compound quantification method of fuzzy information considering both $I_{L}$ metric and $U_{L}$-dispersion. And this method not only contains $I_{L}$-metric method, but it has better interpretability. In practical problems, we can choose different synthesizing effect function to embody different uncertainty conscious in the decision process.

Remark2 Obviously, mode (10) provides a kind of model for describing the sequence feature of fuzzy information $\left(\left(E^{1}, S_{L}\right)\right.$ for short), and it generalizes the existing ranking methods of fuzzy numbers. For example, 1) when $S(x, y)=x,\left(E^{1}, S_{L}\right)$ keeps the order relation $\leq$ defined by level cuts of fuzzy numbers (here $A \leqslant B \quad \Leftrightarrow A_{\lambda} \leqslant B_{\lambda}$ for each $\lambda \in[0,1]$, and $[a, b] \leqslant[c, d] \Leftrightarrow a \leqslant c, b \leqslant d$ ), that is, $\left.S_{L}(A) \leqslant S_{L}(B)\right)$ if $A \leqslant B ; 2$ ) When $S(x, y)=x, L(\lambda) \equiv 1,\left(E^{1}, S_{L}\right)$ just coincides with the order relation proposed in ref.

Remark3 Considering that the fuzzy information is nonnegative in the real problems, or we can realize this feature by an appropriate large positive number, therefore we can set the range of the synthesizing effect function $S(x, y)$ be a map from $[0,+\infty) \times[0,+\infty)$ to $[0,+\infty)$.

Remark4 For the minimum optimization decision problems, we can give the definition of the minimum synthesizing effect function similar to Definition 3, or we can convert it into the maximum optimization decision problems by some methods.

It is easy to verify that, for any given $a, b, \kappa, \beta \in[0,+\infty), S_{1}(x, y)=x(1+a y)^{-b}$, $S_{2}(x, y)=x(1+a)^{-b y}, S_{3}(x, y)=x\left[1+a y\left(1+\kappa \mathrm{e}^{\beta x}\right)^{-1}\right]^{-b}$ are the maximum synthesizing effect function. For mode (6), the above synthesizing effect functions are encouraging punishing state, that is, the uncertainty $U_{L}(A)$ of fuzzy information is applied to the principal value $I_{L}(A)$ by some methods, but the starting point is not same, $S_{1}(x, y)$ and $S_{2}(x, y)$ are with simple punishing mode, no consideration of the dynamic restriction relationship between $I_{L}(A)$ and $U_{L}(A)$, while $S_{3}(x, y)$ considers the dynamic relaxation characteristic of $U_{L}(A)$ based on $I_{L}(A)$, therefore, $a, b$ are parameters reflecting punishment intensity, and $\kappa, \beta$ are reflecting the dynamic requirement of $U_{L}(A)$ based on $I_{L}(A)$.

\section{FCR Assessment Model based on Synthetic Effect}

The main title (on the first page) should begin $13 / 16$ inches ( 7 picas) from the top edge of the page, centered, and in Times New Roman 14-point, boldface type. Capitalize the first letter of nouns, pronouns, verbs, adjectives, and adverbs; do not capitalize articles, coordinate conjunctions, or prepositions (unless the title begins with such a word). Please initially capitalize only the first word in other titles, including section titles and first, second, and third-order headings (for example, "Titles and headings" — as in these guidelines). Leave two blank lines after the title.

\subsection{The General Form of Assessment Model}

In this paper, the general form of the catastrophe risk assessment model can be expressed as follows: 


$$
\begin{aligned}
& \text { Opt } R(k)=F\left(r_{1}^{k}, r_{2}^{k}, \cdots, r_{n}^{k}\right) \\
& \text { (P) } \quad\left\{\begin{array}{l}
r_{i}^{k}=\sum_{j=1}^{m_{i}} u_{i j}^{k} w_{i j} \\
u_{i j}^{k} \geq 0 \\
\sum_{j=1}^{m_{i}} w_{i j}=1, w_{i j} \in[0,1] \\
k=1,2, \cdots, l ; i=1,2, \cdots, n ; \\
j \in m_{i} ; m_{i}=1,2, \cdots
\end{array}\right.
\end{aligned}
$$

Here, $k=1,2, \ldots, l$ is denoted the affected areas, $i=1,2, \ldots, n$ is denoted the type of catastrophe risk assessment indicators in each affected areas, $m_{i}$ is denoted the indicator number of the $i$ th type, $\sum_{j=1}^{m} w_{i j}=1, w_{i j} \in[0,1]$ is denoted the indicators' weight,$u_{i j}^{k}$ is the evaluation result of the kth affected area on the $j$ th indicator inith type, $r_{i}^{k}$ is the comprehensive evaluation results of the $i$ th indicator type on the $k$ th affected area, $O p t R(k)$ is the objective function, and it is denoted the comprehensive evaluation results of the $k$ th affected area. The results are not only denoted the area sort based on risk values, but also the maximum risk value (In this paper, using the former).

\subsection{FCR Assessment Model}

If we can know the weights of the different types on the catastrophe risk indicators, these are $w=\left\{w_{1}, w_{2}, \ldots, w_{n}\right\}, \sum_{i=1}^{n} w_{i}=1, w_{i} \in[0,1]$, and the evaluate values of the single indicators are fuzziness $\tilde{u}_{i j}^{k}$. Then, based on the collation of relevant knowledge about fuzzy numbers, complex quantitative methods and their synthetic effect in the section3, we construct the assessment model of FCR under fuzzy environment as follows:

$$
\begin{aligned}
& \text { OptR } \left.*(k)=\left(\sum_{i=1}^{n} w_{i} S\left(\left(I_{L}\left(\tilde{r}_{i}^{k}\right)\right), U_{L}\left(\tilde{r}_{i}^{k}\right)\right)\right)\right)^{1 / n} \\
& \text { s.t }\left\{\begin{array}{l}
\left.\left.S\left(\left(I_{L}\left(\tilde{r}_{i}^{k}\right)\right), U_{L}\left(\tilde{r}_{i}^{k}\right)\right)\right)=S\left(\left(I_{L}\left(\sum_{j=1}^{m_{i}} u_{i j}^{k} w_{i j}\right)\right), U_{L}\left(\sum_{j=1}^{m_{i}} u_{i j}^{k} w_{i j}\right)\right)\right) \\
\sum_{j=1}^{m_{i}} w_{i j}=1, w_{i j} \in[0,1] \\
\sum_{i}^{n} w_{i}=1, w_{i} \in[0,1] \\
k=1,2, \cdots, l ; i=1,2, \cdots, n \\
j \in m_{i} ; m_{i}=1,2, \cdots
\end{array}\right.
\end{aligned}
$$

$\operatorname{In}\left(P^{*}\right), L_{L}(A)$ is the $I_{L}$-metric of $r_{i}^{k}, U_{L}(A)$ is the $U_{L}$-dispersion of $r_{i}^{k}, S(x, y)$ is the maximum synthesizing effect function, Other parameters are similar in $(p)$.

\section{Comprehensive Assessment of FCR in Shanghai}

Author names and affiliations are to be centered beneath the title and printed in Times New Roman 12-point, non-boldface type. Multiple authors may be shown in a two or three-column format, with their affiliations below their respective names. Affiliations are centered below each author name, italicized, not bold. Include e-mail addresses if possible. Follow the author information by two blank lines before main text. 


\subsection{Index Systems and Weights of the Catastrophe Risk}

In order to verify the feasibility above model, we will assess catastrophe risk in Shanghai area. The catastrophe risks in Shanghai area are more harmful natural disasters including rainstorm, flood, storm surges, tsunami, etc, also including variety of human or social disasters, which are caused by fire, explosion, chemical spills, serious incidents, etc. In this paper, we use the index systems of the catastrophe risks in literature (Yin, 2009) and it was calculated the weight of each indicator by AHP and Delphi Method in the literature, such as shown in Table 2.

In table2,Two indicators are divided into efficiency indicators and cost indicators, efficiency indicators include disastrous factors number (DFN), natural disasters frequency(NDF), secondary disasters frequency(SDF), tolls mortality(TM), tolls injured probability(TIP), disaster affected areas(DAA),economic losses(EL), social impacted level (SIL), economic density(ED), building density(BD), population density(PD), the proportion of people over the 60 age(60APP), lifeline systems density(LSD); Cost indicators include per million hospital beds (PMHB), per capita GDP (PCGDP), emergency evacuation capability(EEC), per capita public green area (PCPGA), warning processing capacity(WPC), the improvement degree of contingency plans (IDCP).

Table 2. The Index Systems and Weights

\begin{tabular}{|c|c|c|c|c|c|}
\hline level indicators & Serial & weight & Two indicators & Serial & weight \\
\hline \multirow{3}{*}{ Disastrous factors } & \multirow{3}{*}{$\mathrm{U}_{1}$} & \multirow{3}{*}{0.085} & DFN & $u_{11}$ & 0.2104 \\
\hline & & & NDF & $u_{12}$ & 0.6537 \\
\hline & & & SDF & $u_{13}$ & 0.1359 \\
\hline \multirow{5}{*}{ History disaster } & \multirow{5}{*}{$\mathrm{U}_{2}$} & \multirow{5}{*}{0.14} & $\mathrm{TM}$ & $u_{21}$ & 0.403 \\
\hline & & & TIP & $u_{22}$ & 0.2083 \\
\hline & & & DAA & $u_{23}$ & 0.0687 \\
\hline & & & EL & $u_{24}$ & 0.2083 \\
\hline & & & SIL & $u_{25}$ & 0.1117 \\
\hline \multirow{5}{*}{ Exposure.Vulnerability } & \multirow{5}{*}{ U3 } & \multirow{5}{*}{0.233} & ED & $u_{31}$ & 0.2324 \\
\hline & & & $\mathrm{BD}$ & $u_{32}$ & 0.1063 \\
\hline & & & PD & $u_{33}$ & 0.3203 \\
\hline & & & 60APP & $u_{34}$ & 0.0553 \\
\hline & & & LSD & $u_{35}$ & 0.2856 \\
\hline \multirow{6}{*}{ Disasterrecoveryforce } & \multirow{6}{*}{$\mathrm{U}_{4}$} & \multirow{6}{*}{0.543} & PMHB & $u_{41}$ & 0.095 \\
\hline & & & PCGDP & $u_{42}$ & 0.2197 \\
\hline & & & EEC & $u_{43}$ & 0.1231 \\
\hline & & & PCPGA & $u_{44}$ & 0.0585 \\
\hline & & & WPC & $u_{45}$ & 0.1845 \\
\hline & & & IDCP & $u_{46}$ & 0.3192 \\
\hline
\end{tabular}

\subsection{Data Acquisition and Analysis of the Assessment Results}

4.2.1. Data Acquisition: Data sources and data quality is the basis of the catastrophe risk assessment, and is a key factor to guarantee the type of disaster and catastrophe risk classification accuracy and objectivity. However, the data of some indicators is not officially announced. In order to ensure data quality, we will divide into five levels on NDF,SDF, TM,,TIP, DAA, EL,,SIL, LSD,EEC, WPC, IDCP, and so on, the data of these indicators is obtained through the survey questionnaire, the other data is obtained by year 
books, the network and literature such as EM-DAT global disaster database, the national Disaster Reduction, Shanghai Municipal Government, Shanghai Municipal Yearbook(2010), Shanghai environment Yearbook(2010). The data is handled as shown appendix-A.

4.2.2. Assessment Results Analysis: According to the above conclusion, first, we deal with data to be standardizing by the formula(6)(the results are omission because the paper length is limit); Taking into account the fuzziness of some assessment indicators, we will use the comprehensive evaluation model $\left(\mathrm{P}^{*}\right)$ of FCR based on synthesizing effect, and obtain risk value and assorting the different areas. Set $L(\lambda)=\lambda, S(x, y)=x(1+a y)^{-b}, a=0.2, b=0.5$, the assessment results and the area sorting based on risk values are shown in table3.

Table 3. The Assessment Results and the Area Sorting based on Risk Values in Shanghai

\begin{tabular}{ccccccc}
\hline $\begin{array}{c}\text { District or County } \\
\text { (D or C) }\end{array}$ & $\boldsymbol{U}_{\mathbf{1}}$ & $\boldsymbol{U}_{\mathbf{2}}$ & $\boldsymbol{U}_{\mathbf{3}}$ & $\boldsymbol{U}_{\mathbf{4}}$ & $\begin{array}{c}\text { Risk values } \\
(\mathbf{R V})\end{array}$ & $\begin{array}{c}\text { Sorting } \\
\text { (DCS) }\end{array}$ \\
\hline Pudong & 0.3124 & 0.387 & 0.127 & 0.164 & 0.6038 & 9 \\
Huangpu & 0.208 & 0.215 & 0.462 & 0.268 & 0.6705 & 1 \\
Luwan & 0.2121 & 0.178 & 0.385 & 0.217 & 0.6392 & 3 \\
Xuhui & 0.2349 & 0.244 & 0.221 & 0.166 & 0.6018 & 10 \\
Changning & 0.2055 & 0.28 & 0.214 & 0.213 & 0.6201 & 8 \\
Jingan & 0.1752 & 0.098 & 0.406 & 0.212 & 0.631 & 6 \\
Putuo & 0.2945 & 0.257 & 0.217 & 0.246 & 0.6379 & 5 \\
Zhabei & 0.2674 & 0.25 & 0.223 & 0.252 & 0.6387 & 4 \\
Hongkou & 0.2646 & 0.224 & 0.326 & 0.282 & 0.6617 & 2 \\
Yangpu & 0.2724 & 0.34 & 0.214 & 0.202 & 0.6254 & 7 \\
Baoshan & 0.2698 & 0.301 & 0.094 & 0.164 & 0.5859 & 14 \\
Minhang & 0.2621 & 0.29 & 0.097 & 0.153 & 0.5796 & 16 \\
Jiading & 0.2411 & 0.178 & 0.059 & 0.215 & 0.5891 & 13 \\
Jinshan & 0.1498 & 0.092 & 0.051 & 0.15 & 0.5322 & 19 \\
Songjiang & 0.1853 & 0.16 & 0.056 & 0.163 & 0.5536 & 18 \\
Qingpu & 0.1651 & 0.133 & 0.052 & 0.208 & 0.5706 & 17 \\
Nanhui & 0.1509 & 0.119 & 0.052 & 0.241 & 0.5856 & 15 \\
Fengxian & 0.1429 & 0.138 & 0.051 & 0.251 & 0.5913 & 12 \\
Chongming & 0.1939 & 0.117 & 0.029 & 0.279 & 0.5998 & 11 \\
\hline & & & & & & \\
\hline
\end{tabular}

In table 3,we shown results as following: 1 the vulnerability exposure index of the catastrophe risk is the most different in Shanghai areas, the values are the highest in Huangpu District, Hongkou District to be up more than 0.65.The reason, there are high population density, aging population dense than the major lifeline systems, regional building coverage, as well as housing construction large proportion in these areas; (2) the change of catastrophe risk level in Shanghai areas is in upper middle range, the highest value in Huangpu District is 0.6705,the lowest value in Jinshan District is 05322; 3 in Shanghai areas, the catastrophe risks are showing a decreasing trend from the center to the periphery, the central urban area risk value is the highest, such as Huangpu District, Hongkou District; in Songjiang and Jinshan District ,the risk value is the lowest. Therefore, this conclusion is consistent with the actual situation in Shanghai areas, and can provide the basis to develop disaster prevention and risk reduction measures for the government. 
Set $L(\lambda)=\lambda, S(x, y)=x(1+a y)^{-b}$, the assessment results and the area sorting based on risk values are shown in table4.Set $L(\lambda)=\lambda^{\tau}$ and $S(x, y)=x(1+a y)^{-b}$, $a=0.5, b=1, \tau$ may take different values, the calculation results are shown in table 5 .

Table 4. The Assessment Results based on the Different Parameters

\begin{tabular}{|c|c|c|c|c|c|c|c|c|}
\hline \multirow{2}{*}{$\begin{array}{c}\text { Parameters } \\
\text { D or C }\end{array}$} & \multicolumn{2}{|c|}{$a=5, b=1$} & \multicolumn{2}{|c|}{$a=0.5, b=1$} & \multicolumn{2}{|c|}{$a=0.5, b=0.005$} & \multicolumn{2}{|c|}{$a=5, b=0.005$} \\
\hline & $\mathrm{RV}$ & DCS & RV & DCS & RV & DCS & RV & DCS \\
\hline Pudong & 0.592 & 9 & 0.603 & 9 & 0.604 & 9 & 0.604 & 9 \\
\hline Huangpu & 0.653 & 1 & 0.669 & 1 & 0.671 & 1 & 0.671 & 1 \\
\hline Luwan & 0.628 & 3 & 0.638 & 3 & 0.64 & 3 & 0.639 & 3 \\
\hline Xuhui & 0.591 & 10 & 0.601 & 10 & 0.602 & 10 & 0.602 & 10 \\
\hline Changning & 0.608 & 8 & 0.619 & 8 & 0.62 & 8 & 0.62 & 8 \\
\hline Jingan & 0.622 & 4 & 0.63 & 6 & 0.631 & 6 & 0.631 & 6 \\
\hline Putuo & 0.62 & 6 & 0.636 & 5 & 0.638 & 5 & 0.638 & 5 \\
\hline Zhabei & 0.621 & 5 & 0.637 & 4 & 0.639 & 4 & 0.639 & 4 \\
\hline Hongkou & 0.64 & 2 & 0.66 & 2 & 0.662 & 2 & 0.662 & 2 \\
\hline Yangpu & 0.614 & 7 & 0.624 & 7 & 0.626 & 7 & 0.626 & 7 \\
\hline Baoshan & 0.576 & 12 & 0.585 & 14 & 0.586 & 14 & 0.586 & 14 \\
\hline Minhang & 0.57 & 15 & 0.579 & 16 & 0.58 & 16 & 0.58 & 16 \\
\hline Jiading & 0.573 & 13 & 0.588 & 13 & 0.59 & 13 & 0.589 & 13 \\
\hline Jinshan & 0.525 & 19 & 0.532 & 19 & 0.532 & 19 & 0.532 & 19 \\
\hline Songjiang & 0.545 & 18 & 0.553 & 18 & 0.554 & 18 & 0.554 & 18 \\
\hline Qingpu & 0.56 & 17 & 0.57 & 17 & 0.571 & 17 & 0.571 & 17 \\
\hline Nanhui & 0.567 & 16 & 0.584 & 15 & 0.586 & 15 & 0.586 & 15 \\
\hline Fengxian & 0.573 & 14 & 0.59 & 12 & 0.592 & 12 & 0.592 & 12 \\
\hline Chongming & 0.58 & 11 & 0.598 & 11 & 0.6 & 11 & 0.6 & 11 \\
\hline
\end{tabular}

Table 5. The Assessment Results based on the Utility Functions of Different Level

\begin{tabular}{ccccccc}
\hline Parameters & \multicolumn{2}{c}{$\tau=1$} & \multicolumn{2}{c}{$\tau=0.5$} & \multicolumn{2}{c}{$\tau=2$} \\
\hline D or C & RV & DCS & RV & DCS & RV & DCS \\
Pudong & 0.603 & 9 & 0.587 & 9 & 0.621 & 9 \\
Huangpu & 0.669 & 1 & 0.652 & 1 & 0.689 & 1 \\
Luwan & 0.638 & 3 & 0.621 & 4 & 0.658 & 3 \\
Xuhui & 0.601 & 10 & 0.585 & 10 & 0.619 & 10 \\
Changning & 0.619 & 8 & 0.602 & 8 & 0.639 & 8 \\
Jingan & 0.63 & 6 & 0.613 & 6 & 0.65 & 6 \\
Putuo & 0.636 & 5 & 0.621 & 5 & 0.654 & 5 \\
Zhabei & 0.637 & 4 & 0.621 & 3 & 0.655 & 4 \\
Hongkou & 0.66 & 2 & 0.644 & 2 & 0.678 & 2 \\
Yangpu & 0.624 & 7 & 0.607 & 7 & 0.644 & 7 \\
Baoshan & 0.585 & 14 & 0.57 & 15 & 0.602 & 13 \\
Minhang & 0.579 & 16 & 0.564 & 16 & 0.596 & 16 \\
Jiading & 0.588 & 13 & 0.575 & 13 & 0.602 & 14 \\
Jinshan & 0.532 & 19 & 0.519 & 19 & 0.546 & 19 \\
Songjiang & 0.553 & 18 & 0.54 & 18 & 0.568 & 18 \\
Qingpu & 0.57 & 17 & 0.556 & 17 & 0.586 & 17 \\
Nanhui & 0.584 & 15 & 0.571 & 14 & 0.599 & 15 \\
Fengxian & 0.59 & 12 & 0.576 & 12 & 0.605 & 12 \\
Chongming & 0.598 & 11 & 0.584 & 11 & 0.614 & 11 \\
\hline
\end{tabular}

In Table 4 and Table 5, According to the model and formula, we set the values of the different parameters and obtained the results of the comprehensive assessment, but these results (comprehensive risk values and regional risk sorting) were not different in general, 
especially, the assessment results in the high-risk areas and low risk areas. For example, the Huangpu District and Hongkou District are belonging to the high-risk areas, but Songjiang and Qingpu District are belonging to the low -risk areas, other areas are a slight change, such as Jiading District, Minhang District and Bashan District. These results indicated that we used fuzzy techniques In the model $(\mathrm{P} *)$, and integrated into the more awareness of man-made, and the assessment results were more robust.

\section{Conclusion and Prospection}

In this paper, according to catastrophe risk characteristics, and the research results for catastrophe risk assessment, we applied fuzzy analysis technique into the comprehensive assessment of catastrophe risk, and had some contributions as following: First, According to the characteristics of catastrophe risks, the fuzzy technique is more conducive to the description and analysis of catastrophic risk. Second, we established the comprehensive evaluation model of FCR based on synthesizing effect, and the model was used to evaluate catastrophe risks including fuzziness indicators. Third, the empirical results indicated that the evaluate risk result is more flexible and more robust when the fuzzy analysis technique is utilized. Without doubt, there are some inadequacy in this paper ,such as the standardized method of multi- attribute indicator system, the synthetic effect function selected and its decision parameters set , and the different methods contrastive analysis, etc.

In the future, the followings will be the aspects researched: First, Indicator system establishment for multi-catastrophe risks or composite catastrophe risk, and the transformation methods and standardized method of multi- attribute indicator system value; Second, The different type synthetic effect functions established, and the different decision parameters selected. Finally, try experimenting with combinations of other fuzzy evaluation model to establish the different type fuzzification models and methods for fuzzy catastrophe risks, and carry comparative studies of these through real cases

\section{Acknowledgements}

This paper is supported by the project from National Key Technologies R \& D Program of China (2012BAH20B03), and Supported by the National Natural Science Foundation of China (71071049,71173129), and Supported by the National Social Science Fund(11AZD011).

\section{References}

[1] T. Ling, "Operation Mode of catastrophe risk Bonds and the Pricing Mechanism", Wuhan: Wuhan University Press (2009).

[2] A. Maskrey, "Disaster Mitigation: A Community Based Approach", Oxford: Oxfam (1989).

[3] H. Zongfu, "Natural disaster risk assessment theory and practice", Beijing: Science Press (2005).

[4] P. Thierry, L. Stieltjes and E. Kouokam, "Multi-hazard risk mapping and assessment on an active volcano: the GRINP project at Mount Cameroon", Natural Hazards, vol. 45, (2008), pp. 429-456.

[5] S. Shaomin, "Disaster Assessment Contents and Methods", vol. 20, (2010), pp.122-130.

[6] L. A. Zadeh, "Fuzzy sets, Information and Control", vol. 8, (1965), pp. 338-353.

[7] H. J. Zimmermann, "Fuzzy set theory and its applications (Second Edition)", London: Kluwer Academic Publishers (1991).

[8] R. Goetschel and W. Voxman, "Topological properties of fuzzy number”, Fuzzy Sets and Systems vol. 10, (1983), pp. 87-99.

[9] P. Diamond and P. Kloeden, "Metric space of fuzzy set: theory and applications", Word Scientific, Singapore (1994).

[10] C. Xiaowei, W. Wenshuang, S. Guibao, and S. Dianyu, "Hybrid multi-attribute decision making based on fuzzy preference relation", Systems Engineering and Electronics, vol. 34, (2012), pp. 529-533.

[11] Z. Hui, Z. Junsheng, C. Longwei and C. Siwei, "Method for Solving a Hybrid Multiple Attribute Decision Making Problem with Improvement”, System Simulation Technology, vol. 5, (2009), pp. 237240. 
[12] L. Fachao, L. Ling, J. Chenxia, W. Ruijiang, and W. Hong, "Research on the 3PL Supplier Selection Based on Set-Valued Satisfaction", Computers and Operations Research, vol. 39, (2012), pp. 18791884.

[13] Y. Zhane, "Research of Urban Natural Disaster Risk Assessment and Case Study", East China Normal University, (2009).

\section{Appendix A}

Table A-1. Original Value of Hazard Indicators in Shanghai

\begin{tabular}{crrr}
\hline D or C & $\boldsymbol{u}_{\mathbf{1 1}}(\boldsymbol{n})$ & $\boldsymbol{u}_{\mathbf{1 2}}(\boldsymbol{\%})$ & $\boldsymbol{u}_{\mathbf{1 3}}(\boldsymbol{\%})$ \\
\hline Pudong & 11 & {$[80,95]$} & {$[80,95]$} \\
Huangpu & 8 & {$[60,75]$} & {$[20,40]$} \\
Luwan & 7 & {$[65,80]$} & {$[20,35]$} \\
Xuhui & 9 & {$[60,80]$} & {$[45,60]$} \\
Changning & 9 & {$[50,60]$} & {$[45,60]$} \\
Jingan & 8 & {$[45,60]$} & {$[20,40]$} \\
Putuo & 11 & {$[85,100]$} & {$[45,50]$} \\
Zhabei & 9 & {$[80,90]$} & {$[40,55]$} \\
Hongkou & 10 & {$[75,80]$} & {$[50,60]$} \\
Yangpu & 10 & {$[70,80]$} & {$[70,80]$} \\
Baoshan & 9 & {$[80,95]$} & {$[40,50]$} \\
Minhang & 10 & {$[75,80]$} & {$[45,60]$} \\
Jiading & 7 & {$[80,90]$} & {$[20,35]$} \\
Jinshan & 7 & {$[40,60]$} & {$[5,20]$} \\
Songjiang & 9 & {$[50,60]$} & {$[20,30]$} \\
Qingpu & 8 & {$[45,60]$} & {$[10,20]$} \\
Nanhui & 7 & {$[40,60]$} & {$[10,15]$} \\
Fengxian & 6 & {$[40,60]$} & {$[5,15]$} \\
Chongming & 6 & {$[65,80]$} & \\
\hline & & & \\
\hline
\end{tabular}

Table A-2. Original Value of Consequence Indicators in Shanghai

\begin{tabular}{crrrrr}
\hline D or C & $\boldsymbol{u}_{2 \mathbf{1}}$ & \multicolumn{1}{c}{$\boldsymbol{u}_{\mathbf{2}}$} & \multicolumn{1}{c}{$\boldsymbol{u}_{\mathbf{2}}$} & $\boldsymbol{u}_{2 \mathbf{s}}$ & \multicolumn{1}{c}{$\boldsymbol{u}_{\mathbf{2}}$} \\
\hline Pudong & Very High & Very High & Very larger & Very High & Very High \\
Huangpu & Low & High & Average & High & High \\
Luwan & Low & Low & Average & High & High \\
Xuhui & middle & middle & Average & High & Very High \\
Changning & middle & Very High & larger & High & High \\
Jingan & Very Low & Very Low & Average & middle & middle \\
Putuo & middle & middle & larger & Very High & High \\
Zhabei & middle & middle & larger & High & Very High \\
Hongkou & middle & Low & larger & High & High \\
Yangpu & High & Very High & Very larger & Very High & High \\
Baoshan & middle & Very High & larger & Very High & Very High \\
\hline
\end{tabular}




\begin{tabular}{|c|c|c|c|c|c|}
\hline D or C & $u_{21}$ & $u_{22}$ & $u_{23}$ & $u_{24}$ & $u_{25}$ \\
\hline Minhang & High & middle & larger & High & Very High \\
\hline Jiading & Low & Low & Average & High & High \\
\hline Jinshan & Very Low & Very Low & Small & Low & Very High \\
\hline Songjiang & Low & Very Low & Average & High & High \\
\hline Qingpu & Low & Very Low & Small & Low & Very High \\
\hline Nanhui & Low & Very Low & Small & Low & middle \\
\hline Fengxian & Low & Low & Small & Low & middle \\
\hline Chongming & Very Low & Very Low & larger & middle & Very High \\
\hline
\end{tabular}

Table A-3. Original Value of Exposure Indicators in Shanghai

\begin{tabular}{|c|c|c|c|c|c|}
\hline D or $\mathbf{C}$ & $u_{31}$ & $u_{32}$ & $u_{33}$ & $u_{34}$ & $u_{35}$ \\
\hline Pudong & 6161.8 & 21.3 & 5355 & 19.4 & High \\
\hline Huangpu & 87302 & 154 & 41459 & 20.9 & Very High \\
\hline Luwan & 58063.8 & 158 & 33590 & 22.9 & Very High \\
\hline Xuhui & 24310.5 & 78 & 17571 & 21.6 & High \\
\hline Changning & 21810.3 & 77.4 & 17073 & 20.4 & High \\
\hline Jingan & 62976.5 & 199 & 33793 & 23.3 & Very High \\
\hline Putuo & 14895 & 83.4 & 20447 & 20.4 & High \\
\hline Zhabei & 19100.8 & 89.5 & 25666 & 20.3 & middle \\
\hline Hongkou & 43204.7 & 125 & 33352 & 21.4 & High \\
\hline Yangpu & 17742 & 70.6 & 19480 & 19.7 & High \\
\hline Baoshan & 2288.4 & 13 & 4897 & 19.3 & middle \\
\hline Minhang & 2906.8 & 19.4 & 4922 & 19.4 & middle \\
\hline Jiading & 814.4 & 9.8 & 2111 & 21.1 & Low \\
\hline Jinshan & 624.4 & 2.8 & 1083 & 18.5 & Low \\
\hline Songjiang & 668 & 10.7 & 1558 & 18.7 & Low \\
\hline Qingpu & 408.6 & 4.7 & 1111 & 19.4 & Low \\
\hline Nanhui & 622.3 & 3.1 & 1373 & 18.1 & Low \\
\hline Fengxian & 403.4 & 2.3 & 1085 & 18.8 & Low \\
\hline Chongming & 283.2 & 0.7 & 566 & 22.5 & Very Low \\
\hline
\end{tabular}

Table A-4. Original Value of Resilience Indicators in Shanghai

\begin{tabular}{crrrrrr}
\hline D or C & \multicolumn{1}{c}{$\boldsymbol{u}_{\mathbf{4 1}}$} & \multicolumn{1}{c}{$\mathbf{u}_{\mathbf{4 2}}$} & \multicolumn{1}{c}{$\boldsymbol{u}_{\mathbf{4 3}}$} & \multicolumn{1}{c}{$\boldsymbol{u}_{\mathbf{4 4}}$} & \multicolumn{1}{c}{$\boldsymbol{u}_{\mathbf{4 5}}$} & \multicolumn{1}{c}{$\boldsymbol{u}_{\mathbf{4 6}}$} \\
\hline Pudong & 26.1 & 17500 & middle & 24.2 & Very Good & High \\
Huangpu & 101.3 & 18000 & Low & 1.37 & Average & Low \\
Luwan & 154.3 & 14900 & Low & 1.56 & Good & middle \\
Xuhui & 128.5 & 15000 & middle & 4.63 & Good & middle \\
Changning & 49.9 & 13600 & Low & 6.73 & Very Good & middle \\
Jingan & 175.1 & 15500 & Low & 0.94 & Good & High \\
Putuo & 40.1 & 9500 & middle & 5.15 & Average & Low \\
Zhabei & 61.7 & 8000 & middle & 2.81 & Average & Low \\
Hongkou & 86 & 12890 & Low & 1.82 & Poor & Low \\
\hline
\end{tabular}




\begin{tabular}{crrrrrr}
\hline D or C & $\boldsymbol{u}_{\mathbf{4 1}}$ & $\mathbf{u}_{\mathbf{4 2}}$ & $\boldsymbol{u}_{\mathbf{4 3}}$ & $\boldsymbol{u}_{\mathbf{4 4}}$ & \multicolumn{1}{c}{$\boldsymbol{u}_{\mathbf{4 5}}$} & $\boldsymbol{u}_{\mathbf{4 6}}$ \\
\hline Yangpu & 49.5 & 10000 & Low & 3.74 & Good & Very High \\
Baoshan & 32.6 & 7600 & High & 18.9 & Good & Very High \\
Minhang & 29.4 & 12600 & High & 24.71 & Very Good & High \\
Jiading & 26.4 & 7100 & High & 23.59 & Poor & middle \\
Jinshan & 50.5 & 7000 & Very High & 15.48 & Good & Very High \\
Songjiang & 41.2 & 7600 & High & 11.31 & Average & Very High \\
Qingpu & 24 & 6000 & Very High & 29.42 & Good & middle \\
Nanhui & 51.1 & 5800 & Very High & 11.57 & Average & Low \\
Fengxian & 61.7 & 5400 & High & 13.46 & Average & Low \\
Chongming & 53.7 & 4800 & middle & 10.5 & Average & Low \\
\hline
\end{tabular}

\section{Author}

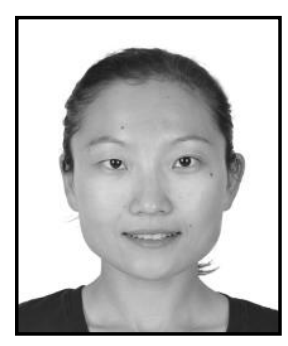

Xiaoxia Zhu, She is a teacher of Hebei University of Science and Technology. She has worked in the university since 2004. Her research and teaching areas include fuzzy decision-making theory, fuzzy risk, fuzzy control theory, etc. She has finished many research programs in the national and provincial level, e.g. the National Science-supporting program, and published many articles on International meetings, such as LISS, ICMLC, ICNC-FSKD etc, and important journals, such as Computer Science, Industrial Engineering and Management, etc. 
International Journal of Security and Its Applications

Vol.9, No.8 (2015) 